\title{
Nano bubble-Size dependence of surface tension and inside pressure
}

$\operatorname{AUTHOR}(S)$ :

Matsumoto, Mitsuhiro; Tanaka, Kotaro

\section{CITATION:}

Matsumoto, Mitsuhiro ...[et al]. Nano bubble-Size dependence of surface tension and inside pressure. Fluid Dynamics Research 2008, 40(7-8): 546-553

\section{ISSUE DATE:}

2008-07-30

URL:

http://hdl.handle.net/2433/91245

\section{RIGHT:}

This is an author-created, un-copyedited version of an article accepted for publication in Fluid Dynamics Research. IOP Publishing Ltd is not responsible for any errors or omissions in this version of the manuscript or any version derived from it. The definitive publisher authenticated version is available online at 10.1016/j.fluiddyn.2007.12.006; This is not the published version. Please cite only the published version.; この論文は出版社版でありません。引用の際には出版社 版をご確認ご利用ください。 


\title{
Nano Bubble \\ - Size Dependence of Surface Tension and Inside Pressure -
}

\author{
Mitsuhiro Matsumoto* and Kotaro Tanaka \\ Department of Mechanical Science and Engineering, \\ Graduate School of Engineering, Kyoto University, \\ Yoshida-honmachi, Kyoto 606-8501, Japan
}

\begin{abstract}
The Young-Laplace (Y-L) equation describes the difference between inside pressure and outside pressure of a spherical bubble due to surface tension. The Y-L equation is simply deduced from mechanical stability of a bubble, but it is still controversial whether the Y-L equation can be used for tiny bubbles, such as a "nano bubble", because the pressure difference divergently increases as the bubble radius $R$ decreases. We investigated a spherical vapor bubble in Lennard-Jones liquid with molecular dynamics simulation, mainly looking into its mechanical stabilities. We generated a tiny bubble of various size ( $R \simeq 1.7 \mathrm{~nm}-5 \mathrm{~nm}$ in argon unit) under equilibrium conditions by changing the simulation cell size and the number of molecules. The liquid pressure was evaluated with the virial expression, which was negative in general and was found to be strongly dependent on $R$. The vapor pressure was estimated from the vapor density via an empirical equation of state. The vapor pressure was found to be independent of $R$ and very close to the vapor pressure at bulk liquid-vapor equilibrium. Then we assumed the Y-L equation to calculate the surface tension of the bubble, which turned out to be also independent of $R$. Thus we confirm that the Y-L equation is valid even for nano-scale bubbles.
\end{abstract}

Key words: Nano bubble, Surface tension, Young-Laplace equation, Vapor pressure, Molecular simulation

* Email address: matsumoto@kues.kyoto-u.ac.jp 


\section{Introduction}

Micro bubbles recently gain much attention in wide range of fields. In particular, bubbles with size of sub-micrometer, or "nano bubbles", have become an attractive research target, partly because of recent technological progress of micro bubble generations [e.g., Takahashi et al. (2003)], and partly in prospect of their fruitful applications, such as MEMS devices and biological treatments. However, experimental investigation of their physical properties is far behind the applications due to their size and fragileness.

In this paper, we focus on the mechanical stability of a single spherical nano bubble. The force balance concerning a spherical bubble of radius $R$ is usually described by the Young-Laplace (Y-L) equation,

$$
P_{v a p}=P_{l i q}+\frac{2 \gamma}{R}
$$

Here, $P_{\text {vap }}$ and $P_{l i q}$ are the pressure inside (vapor phase) and outside (liquid phase) of the bubble, respectively, and $\gamma$ is the surface tension. As far as the bubble size is micro- or milli-meter order, the Y-L equation satisfactorily explains the bubble dynamics [Skripov (1974); Brennen (1995)]. The question here arising is how far the Y-L equation is applicable for nano bubbles. When the bubble size decreases to an order of sub-micrometer, the pressure difference $\Delta P \equiv P_{\text {vap }}-P_{\text {liq }}$ increases as an inverse power of $R$. For example, a bubble of $R=10 \mathrm{~nm}$ in water at room temperature $(\gamma \simeq 0.073 \mathrm{~N} / \mathrm{m})$ will show $\Delta P \simeq 1.5 \times 10^{7} \mathrm{~Pa}$, or $150 \mathrm{~atm}$. How can this tiny bubble be mechanically stable under atmospheric pressure?

There are two possibilities to solve this difficulty. The first one is to abandon the Y-L equation, assuming that it is applicable only in macroscopic or continuum level, as pointed out by Nagayama et al. (2006). In this case, we will need some replacing equation based on some atomic scale structure of bubble surface. The second is that the Y-L equation is still applicable for nano bubbles, but the surface tension much differs from the value of macroscopic system, i.e., flat surface. The curvature dependence of surface tension and vapor pressure has been well investigated for droplets [Tolman (1949); Thompson et al. (1984); Koga et al. (1998); Yaguchi et al. (2007)], and it is generally concluded that the surface tension of a droplet decreases as the size decreases. We then naïvely expect that the surface tension of a tiny bubble is much smaller than the bulk value, which may reconcile the Y-L equation and the nano bubble stability. However, Park et al. (2001) recently reported with a molecular simulation that the surface tension of a microbubble rather increases slightly with the decrease of its radius, which makes the reconciliation even more difficult.

Our approach in this paper is the second one. We make a series of molecular 
simulations to evaluate both $P_{l i q}$ and $P_{v a p}$ of a tiny bubble with various size. Then by assuming Eq. (1), we obtain the surface tension, investigate its size dependence, and look into the stability mechanism.

\section{Method}

\subsection{System}

To save computational resources, we focus on a system of monatomic simple liquid. Many particles are confined in a cubic cell with three-dimensional periodic boundary conditions. Two systems, the number of particles $N=25,000$ and 125,000 , are used. The Lennard-Jones (12-6) potential is adopted as the particle-particle interaction:

$$
\phi_{L J}(r)=4 \epsilon\left[\left(\frac{\sigma}{r}\right)^{12}-\left(\frac{\sigma}{r}\right)^{6}\right]
$$

where $r$ is the distance between the particles, and $\sigma$ and $\epsilon$ are the size and the energy parameters, respectively. When we regard this system as argon liquid, the parameters correspond to $\sigma \simeq 0.34 \mathrm{~nm}$ and $\epsilon \simeq 1.67 \times 10^{-21} \mathrm{~J}$. In this paper, physical quantities are expressed in dimensionless unit, reduced by $\sigma$, $\epsilon$, and the particle mass $m$. The interaction is simply truncated at $r / \sigma=3.5$. To avoid irrelevant dependence on the system size, no long-range corrections are done.

\subsection{Molecular Dynamics Simulation}

We carry out canonical ensemble ( $N, V, T$ constant) molecular dynamics (MD) simulation of the liquid system. First, the system is equilibrated at a chosen temperature $T$ with volume $V$ appropriate to a normal liquid state. Then, a repulsive external force field is applied at the central part of the cell to generate a tiny vacancy, or a "bubble". After the equilibrium is reached, the force field is removed. With varying the system volume $V$, we can obtain liquid system containing a single bubble of various size. In this work, we compare a system with $k_{B} T / \epsilon=0.7$ ( $k_{B}$ is the Boltzmann constant), which is close to the triple point temperature, and a system with higher temperature $k_{B} T / \epsilon=$ 1.0. An example of snapshot is given in Fig. 1, where a slice of the whole system is shown. Several particles are seen inside the bubble, which evaporated spontaneously. Therefore, the realized is a "vapor bubble," inside of which is not the vacuum. 


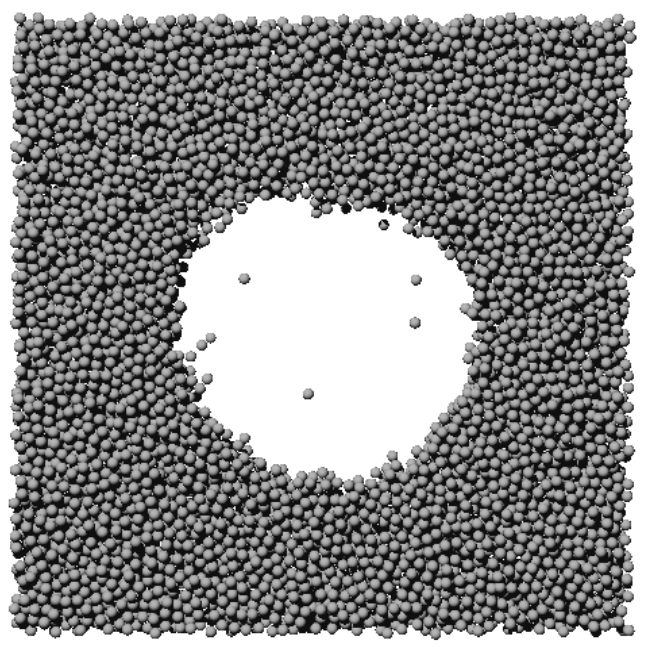

Fig. 1. Snapshot of the bubble system near the triple point temperature; a slice containing the bubble is shown.

Sufficiently long MD simulation (more than $1 \mathrm{~ns}$ in argon unit) is done at the equilibrium to accumulate the particle positions and the system pressure for data analyses.

\section{Results}

\subsection{Density Profile}

A tiny bubble easily migrates in the simulation cell. To determine the bubble position, we divide the cell into small meshes (mesh size $=1.5 \sigma)$, and regard the assembly of vacant mesh points as the "bubble"; more rigorous definitions have been proposed by Kinjo et al. (1998) and Kikugawa et al. (2007). The bubble position $\boldsymbol{r}_{0}$ is defined as the "center of mass" of the bubble.

The density profile $n(r)$ is the average number density at distance $r$ from $\boldsymbol{r}_{0}$; here we assume the spherical symmetry. Results of $n(r)$ at $T=0.7$ are shown in Fig. 2.

The density profile is well fitted to the following hyperbolic function:

$$
n(r)=\frac{n_{l i q}+n_{\text {vap }}}{2}+\frac{n_{\text {liq }}-n_{\text {vap }}}{2} \tanh \left(\frac{r-R}{w}\right)
$$

where the liquid density $n_{l i q}$, the vapor density $n_{\text {vap }}$, the bubble radius $R$, and the width of interface $w$ are the fitting parameters. We found that $w \simeq$ $0.8-1.0 \sigma$ at $T=0.7$ and $\simeq 1.8-2.0 \sigma$ at $T=1.0$, almost independent of $R$. However, $n_{\text {liq }}$ slightly decreases with the decrease of $R$, which means that the 


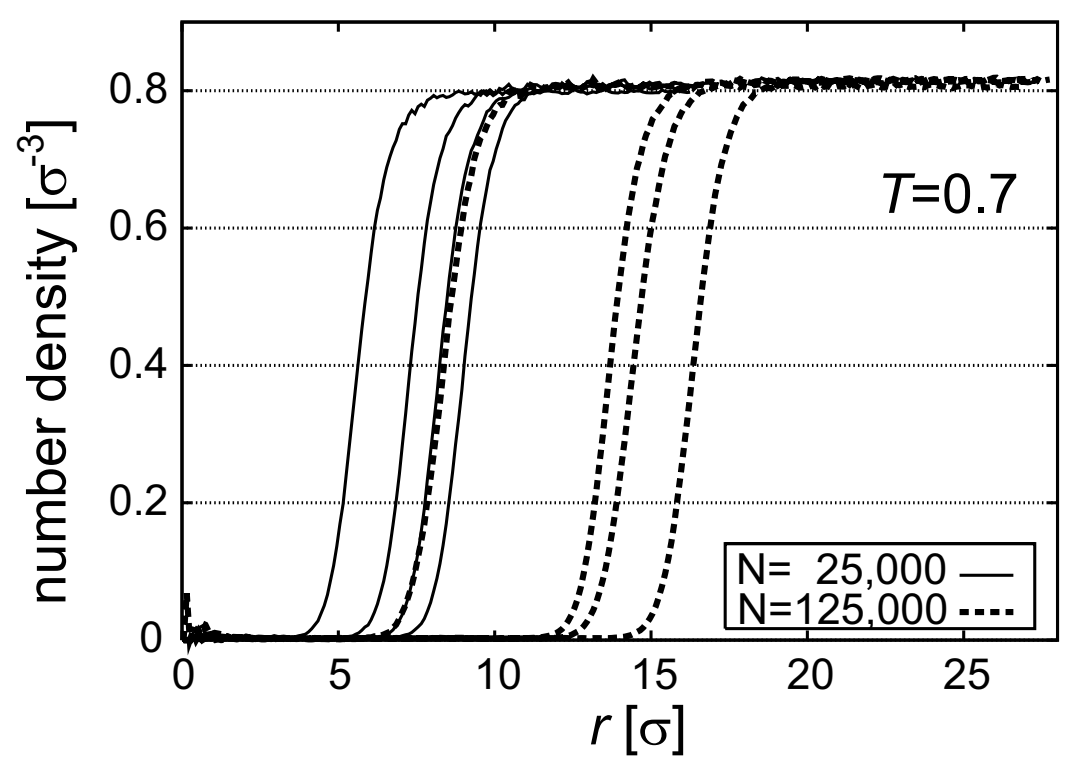

Fig. 2. Density profile from the bubble center: at low temperature.

liquid is "stretched" due to the surface tension, as will be discussed in the next section.

\subsection{Liquid Pressure}

In this work, the pressure is calculated via a usual virial expression:

$$
P=\frac{N}{V} k_{B} T-\frac{1}{6 V}\left\langle\sum_{i j} \boldsymbol{r}_{i j} \cdot \frac{\partial \phi_{L J}\left(r_{i j}\right)}{\partial \boldsymbol{r}_{i j}}\right\rangle
$$

When Eq. (4) is applied to the "bulk" liquid region, i.e., the region far from the bubble, we can obtain the liquid pressure $P_{l i q}$. However, $P$ evaluated with the whole system was found to be almost equal to $P_{l i q}$ because the contribution of the bubble region is much smaller than the liquid region. The difference between $P$ (whole system) and $P_{l i q}$ is much less than $1 \%$.

The results are shown in Fig. 3. The liquid pressure in general takes a negative value, which means that the liquid is in a stretched state. As the bubble size decreases, the liquid becomes more stretched due to the surface tension, which causes the decrease of the number density as in Fig. 4, and $P_{\text {liq }}$. 


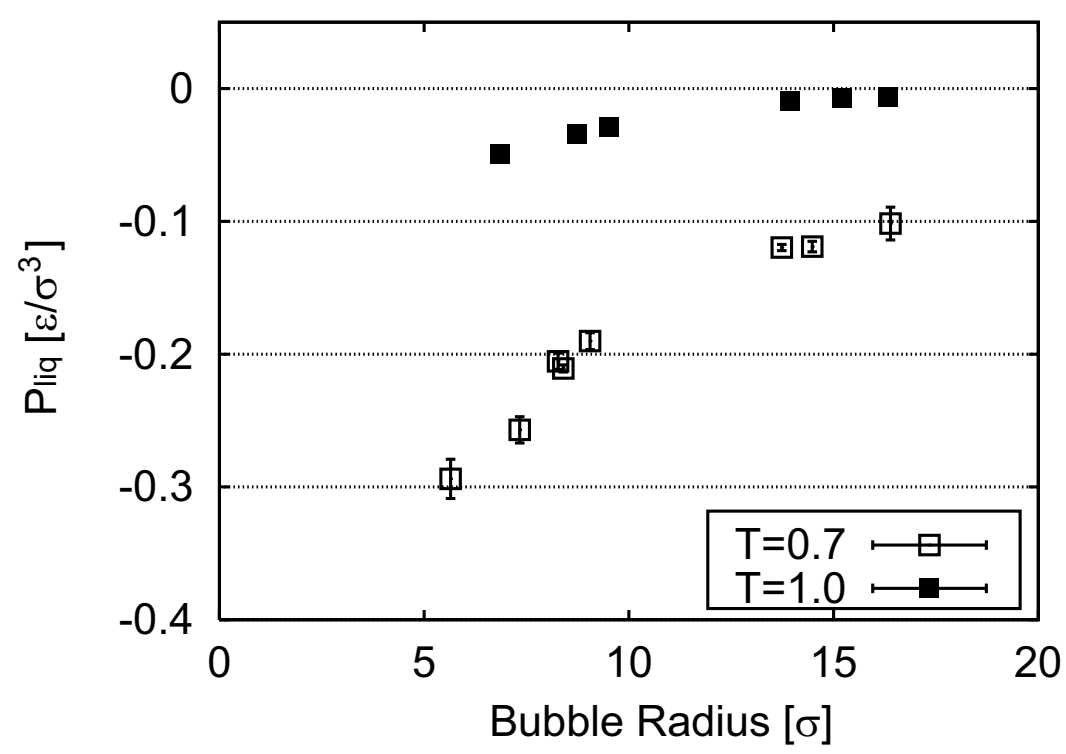

Fig. 3. Bubble size dependence of the pressure in the surrounding liquid. The error bars show the standard deviation (SD), which is estimated by divided into the total simulation run of more than 1 ns into several parts of 200 ps. Typical SD is 0.008 at $T=0.7$ and 0.004 at $T=1.0$.

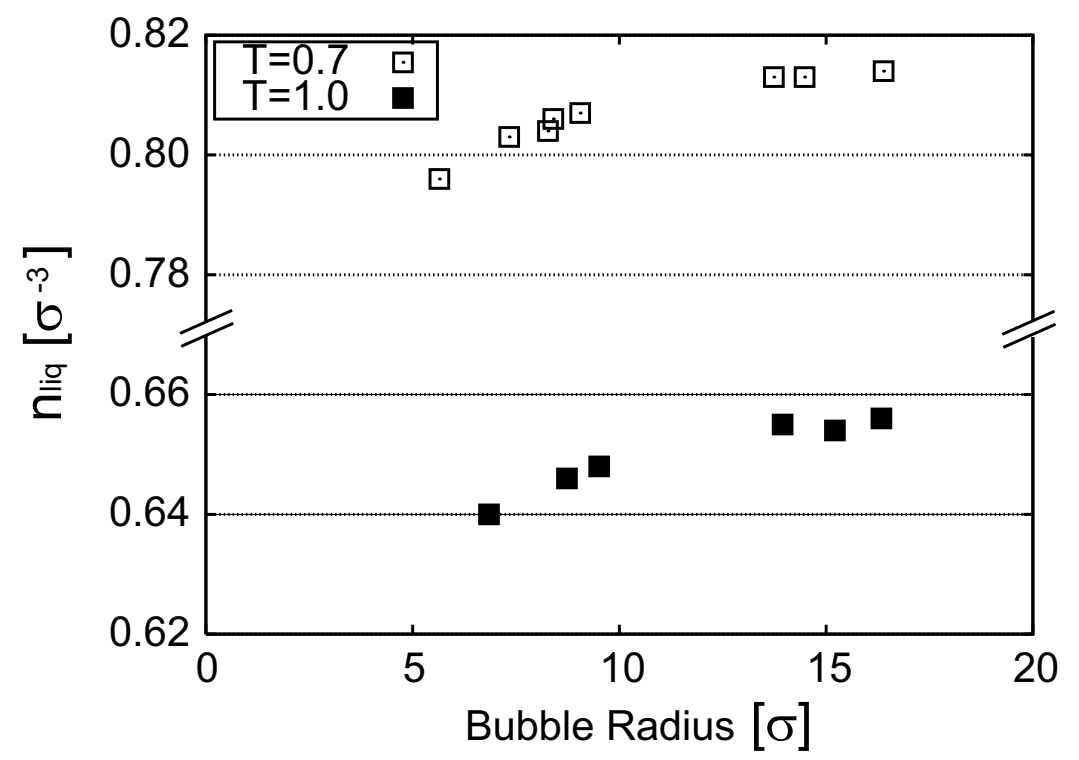

Fig. 4. Bubble size dependence of the number density of the surrounding liquid. The standard deviation, estimated similarly as in Fig. 3, is too small to be shown in the figure, typically less than 0.002 .

\subsection{Vapor Pressure}

In principle, the vapor pressure $P_{v a p}$ is calculated with Eq. (4) applied to the inside region of the bubble. However, this is practically impossible because the 
bubble is too small, the density of vapor is too low, and the statistics is too poor to obtain a precise value with the virial expression. We take another approach to evaluate $P_{v a p}$, which is through the vapor density $n_{\text {vap }}$. We carried out separate MD simulations for bulk vapor phase to make an empirical equation of state $\left(n_{v a p}\right.$ dependence of $P_{v a p}$ at given $T$ ). By evaluating $n_{v a p}$ inside the bubble and comparing it with the equation of state, $P_{v a p}$ inside was satisfactorily obtained. Note that $n_{\text {vap }}$ is calculated from direct count of particles inside the bubble, not from the fitting parameters of $n(r)$ [Eq. (3)], which is much less precise.

The results are shown in Fig. 5. $P_{\text {vap }}$ at $R \rightarrow \infty$ is the vapor pressure on a flat surface, or the saturated vapor pressure in bulk, which was evaluated from separate MD simulations of a flat liquid layer. It is amazing that $P_{v a p}$ is almost independent of the bubble size and agrees with the bulk (macroscopic) value.

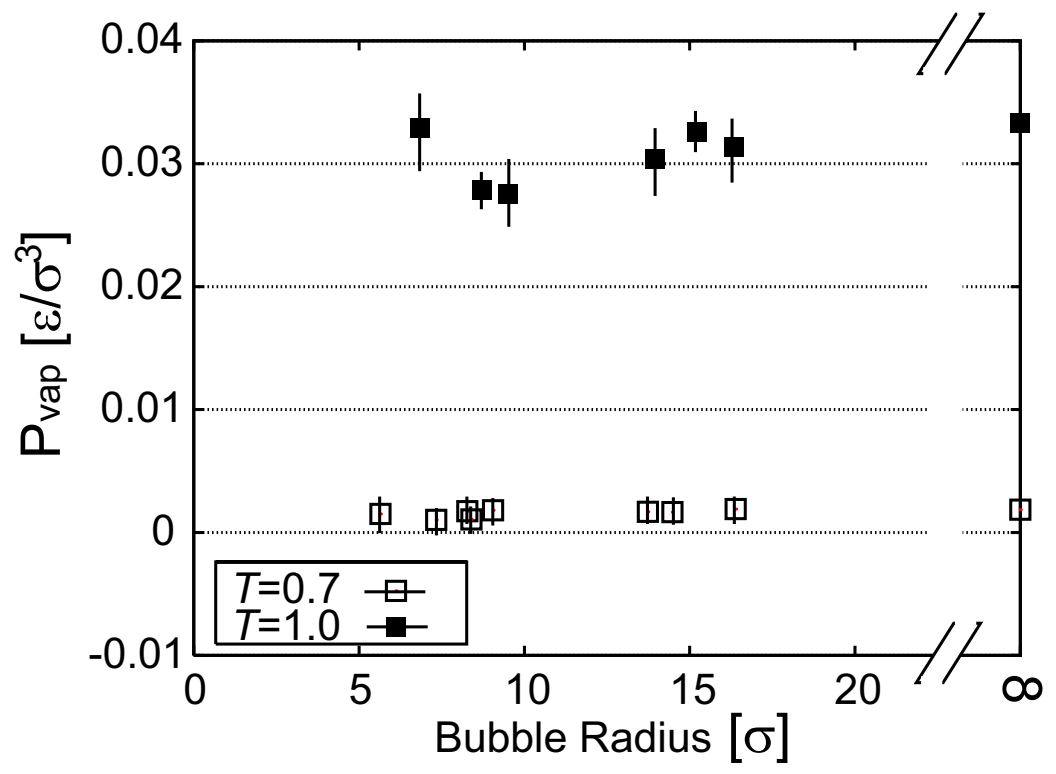

Fig. 5. Bubble size dependence of the pressure inside the bubble. Typical standard deviation, estimated by divided into the total simulation run of more than $1 \mathrm{~ns}$ into several parts of $400 \mathrm{ps}$, is 0.001 at $T=0.7$ and 0.003 at $T=1.0$.

\subsection{Surface Tension}

Now that both $P_{l i q}(R)$ and $P_{\text {vap }}(R)$ are known, we can estimate the $R$ dependence of the surface tension $\gamma$, assuming the Y-L equation, Eq. (1).

Figure 6 shows the results; the value at $R \rightarrow \infty$ is directly obtained from the separate MD simulation of a flat surface, as the difference of pressure tensor 
components [Rowlinson and Widom (1982)]. The surface tension is amazingly constant even when the bubble is as small as atomic scale; note that $R=5 \sigma$ corresponds to $\sim 1.7 \mathrm{~nm}$ in argon unit.

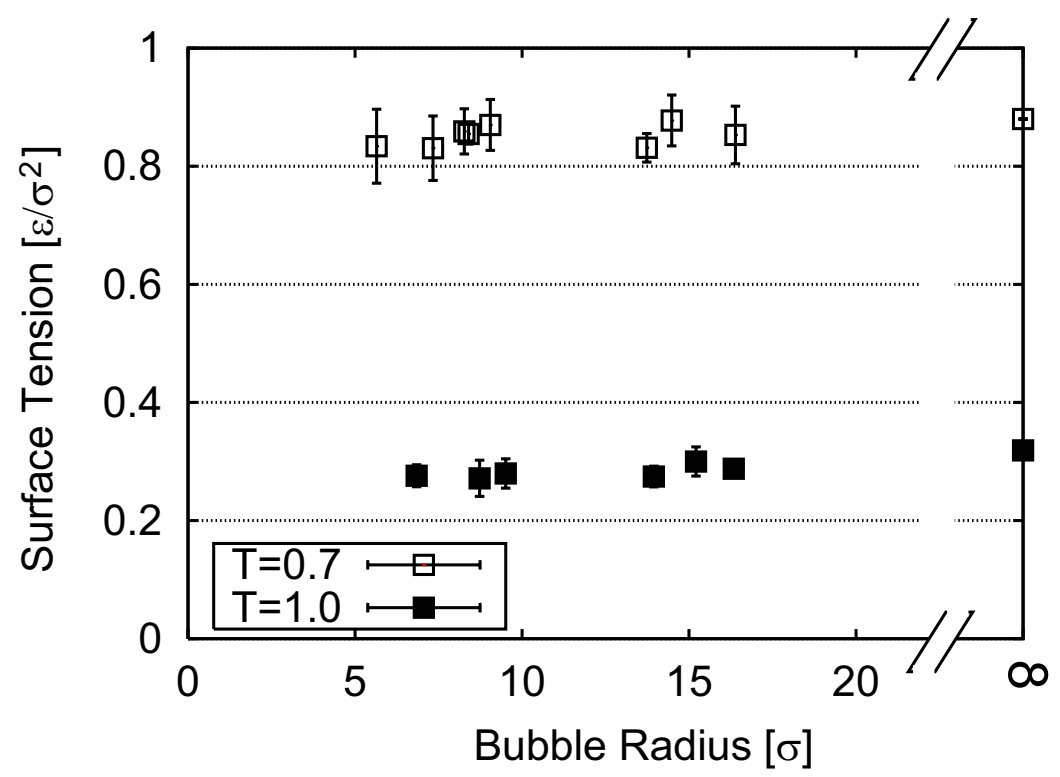

Fig. 6. Bubble size dependence of the surface tension. The error bars show the uncertainty caused by the standard deviations of pressure shown in Figs. 2 and 5.

Our results of $R$ independent $\gamma$ seems different from those by Park et al. (2001), who conclude that $\gamma$ of a tiny bubble slightly increases (up to $\sim 15 \%$ ) from the bulk surface tension. There are two possible reasons. The first is the difference of method to evaluate $\gamma$. While Park et al. (2001) directly calculated the local pressure tensor, we focus on the difference of $P_{l i q}$ and $P_{\text {vap }}$ assuming the Y-L equation. In general, their method is more preferable because it does not need any further assumption, but the local pressure tensor for a tiny bubble can be largely fluctuating due to its strong inhomogeneity and extreme locality. There may also be a problem of virial evaluation on curved surfaces [Ikeshoji et al. (2003)]. The second is the difference of system size. Sufficiently large system is required to realize the bulk liquid region around the bubble. As already pointed out by them, their density and pressure profiles in the liquid region seem to have non-zero gradients, which may cause some errors. Even with these differences, we may conclude that the curvature dependence of $\gamma$ of a tiny bubble is much smaller, if exists, than that of a droplet.

\section{Conclusion}

We investigated the size dependence of vapor bubble properties using molecular dynamics simulations of Lennard-Jones model liquid, and found that 
(1) The vapor density and the vapor pressure inside the bubble is independent of the bubble radius, equal to those of the saturated vapor in bulk equilibrium.

(2) The liquid surrounding the bubble is at a strongly stretched state. As the bubble size decreases, the liquid is exposed to more tensile stress.

(3) The surface tension evaluated with assumption of the Y-L equation is also little dependent on the bubble radius, and agrees with the surface tension of a planer interface.

Now we turn to the mechanical stability of nano bubbles. When we admit that Eq. (1) is valid even at nano scale, and also that $P_{\text {vap }}$ and $\gamma$ equal to respective bulk values, stable nano bubbles can exist only in liquid under highly tensile stress, or large negative pressure. Thus we are forced to conclude that nano bubbles observed under atmospheric pressure are either at some non-equilibrium state (e.g., shrinking with time) or containing some impurity, such as non-condensable gas or adsorbed contamination.

Strictly speaking, the above conclusion is for the Lennard-Jones liquid, which has only short-range particle interactions. During this work, we tried hard to generate a tinier bubble (e.g., $R<3 \sigma$ ) but failed; after a long MD run (e.g., $1 \mathrm{~ns}$ in argon unit), such bubbles eventually collapsed. Attractive interaction between particles seems to cause this instability. Considering that the interaction is truncated at $r=3.5 \sigma$, it is suspected that curvature effects, if exist, may appear for bubbles of size comparable with the particle interaction range. Wider interaction range may change the situation. With this in mind, similar simulatoins for a nano bubble in water, which has long-ranged Coulombic interactions between atoms, are currently under way.

\section{Acknowledgments}

The authors are grateful to Prof. K. Yasuoka at Keio University and Prof. X. C. Zeng at University of Nebraska-Lincoln for stimulating discussion, and Dr. H. Yaguchi and Prof. S. Fujikawa at Hokkaido University for providing their recent results on droplets. Financial support by the 21st century COE program (Kyoto University), "Research and Education on Complex Functional Mechanical Systems," is greatly acknowledged.

\section{References}

Brennen, C. E. 1995. Cavitation and Bubble Dynamics. Oxford University Press. 
Ikeshoji, T., Hafskjold, B., and Furuholt, H. 2003. Molecular-level calculation scheme for pressure in inhomogeneous systems of flat and spherical layers. Molecular Simulation, 29, 101-109.

Kikugawa, G., Takagi, S., and Matsumoto, Y. 2007. A molecular dynamics study on liquid-vapor interface adsorbed by impurities. Computers and Fluids, 36, 69-76.

Kinjo, T., and Matsumoto, M. 1998. Cavitation processes and negative pressure. Fluid Phase Equilibria, 144, 343-350.

Koga, K., Zeng, X. C., and Shchekin, A. K. 1998. Validity of Tolman's equation: how large should a droplet be? J. Chem. Phys., 109, 4063-4070.

Nagayama, G., Tsuruta, T., and Cheng, P. 2006. Molecular dynamics simulation on bubble formation in a nanochannel. Int. J. Heat and Mass Transf., $494437-4443$.

Park, S. H., Weng, J. G., and Tien, C. L. 2001. A molecular dynamics study on surface tension of microbubbles. Int. J. Heat and Mass Transfer, 44, $1849-1856$.

Rowlinson, J. S. and Widom, B. 1982. Molecular Theory of Capillarity. Clarendon Press.

Skripov, V. P., 1974. Metastable Liquids. John Wiley and Sons.

Takahashi, M., Kawamura, T., Yamamoto, Y., Ohnari, H., Himuro, S., and Shakkutsui, H. 2003. Effect of Shrinking Microbubble on Gas Hydrate Formation. J. Phys. Chem. C, 107, 2171-2173.

Thompson, S. M., Gubbins, K. E., Walton, J. P. R. B, Chantry, R. A. R., and Rowlinson, J. S. 1984. A molecular-dynamics study of liquid-drops. J. Chem. Phys., 81, 530-542.

Tolman, R. C. 1949. The effect of droplet size on surface tension. J. Chem. Phys., 17, 333-337.

Yaguchi, H., Yano, T., and Fujikawa, S. 2007. Molecular Dynamics Study of Evaporation of Nanodroplets. Proc. Int. Conf. Multiphase Flow, S4-Thu-D59. 